\title{
Stock assessment based on body size information
}

\author{
YUJI UEDA, AND TAKASHI MATSUISHI \\ Graduate School of Fisheries Sciences, Hokkaido University, Hakodate 041-8611, Japan \\ (ueda@fish.hokudai.ac.jp)
}

\section{KEY WORDS: Pacific cod, SPR, stock assessment, walleye pollock, weight, YPR}

\section{INTRODUCTION}

The most common methods currently used in fisheries stock assessment rely on catch-at-age data. ${ }^{1}$ This is used for virtual population analysis. YPR and SPR are mostly calculated based on age at first capture. Thus the age information of fish is important.

However, maturity, selectivity, price class, etc. depend on length or weight rather than age.

Matsuishi ${ }^{2}$ and Ueda et al. ${ }^{3}$ developed the size-based virtual population analyses, LPA and WPA, using catch-at-size and size transition matrixes. Using the size transition matrix, we can also calculate the size-based YPR and SPR. Generally, catch-at-size (specially -weight) is easily obtained and this is one of merits of using size-based methods.

In this presentation we introduce the weight-based yield per recruit (YPR/W) and spawning biomass per recruit (SPR/W) analyses, and applied these to Pacific cod and walleye pollock off the Pacific coast of southern Hokkaido (PCSH), Japan.

\section{MATERIALS AND METHODS}

To compose the "forward" weight transition matrix $\left(\mathbf{P}_{f}\right)$, we assume that the weight of fishes at year $j W_{j}$ is normally distributed at year $j+1$ with average weight $w_{j+1}$ and standard deviation $\sigma_{w}$. Components $(k, i)$ of $\mathbf{P}_{f}, p_{k i}$ represent the probability that a fish in a weight class $k$ at year $j$ belongs to a weight class $i$ at year $j+1$.

To calculate the YPR/W and SPR/W, we put the population number recruited in the first year in the smallest weight class, as 1 . For this recruited year class, we calculate the population number after the second year in weight class $i$ using Eq. (1):

$N_{i, j+1}=e^{-\left(s_{i}+M\right)} \sum_{k=1}^{K}\left(p_{k, i} N_{k, j}\right)$

where $f$ is fishing mortality, $s_{i}$ is selectivity in weight class $i$, and $M$ is natural mortality.

For example, the population number derived using Eq. (1) is shown in Table 1.
Table 1 An example of the transition of population number.

\begin{tabular}{crrrrrrrrrrr}
\hline $\begin{array}{l}\text { Weight } \\
\text { class } i(\mathrm{~kg})\end{array}$ & 1 & \multicolumn{1}{c}{ Year $j$} & \multicolumn{1}{c}{3} & \multicolumn{1}{c}{5} & \multicolumn{1}{c}{6} & 7 & 8 & 9 & 10 \\
\hline $0-1$ & 1.00 & 0 & 0 & 0 & 0 & 0 & 0 & 0 & 0 & 0 \\
$1-2$ & 0 & 0.82 & 0.01 & 0 & 0 & 0 & 0 & 0 & 0 & 0 \\
$2-3$ & 0 & 0 & 0.63 & 0.04 & 0 & 0 & 0 & 0 & 0 & 0 \\
$3-4$ & 0 & 0 & 0.03 & 0.43 & 0.05 & 0.01 & 0 & 0 & 0 & 0 \\
$4-5$ & 0 & 0 & 0 & 0.07 & 0.17 & 0.04 & 0.01 & 0 & 0 & 0 \\
$5-6$ & 0 & 0 & 0 & 0 & 0.05 & 0.07 & 0.02 & 0.01 & 0 & 0 \\
$6-7$ & 0 & 0 & 0 & 0 & 0.01 & 0.03 & 0.03 & 0.01 & 0.01 & 0 \\
$7-8$ & 0 & 0 & 0 & 0 & 0 & 0 & 0.01 & 0.01 & 0.01 & 0.01 \\
\hline
\end{tabular}

This example is calculated using $f=0.5, s_{i}=0$ (from $0-1 \mathrm{~kg}$ to $2-3 \mathrm{~kg}$ weight classes), $s_{i}=1$ (from $3-4 \mathrm{~kg}$ to $7-8 \mathrm{~kg}$ weight classes) and $M=0.2$.

YPR/W is calculated as Eq. (2):

$\mathrm{YPR} / \mathrm{W}=\sum_{j=1}^{\prime} \sum_{i=1}^{l}\left\{w_{i} \hat{N}_{i, j}\left(1-e^{-\left(f s_{i}+M\right)}\right) \frac{f s_{i}}{\left(f s_{i}+M\right)}\right\}$

where $w_{i}$ is the midpoint of weight class $i$.

$\mathrm{SPR} / \mathrm{W}$, is calculated as Eq. (3):

$\mathrm{SPR} / \mathrm{W}=\sum_{j=1}^{J} \sum_{i=1}^{I} w_{i} N_{i, j} m_{i}$

where $m_{i}$ is the maturity rate of weight class $i$.

For both species of Pacific cod and walleye pollock off the PCSH, we calculated YPR/W, \%SPR/W, age-based YPR and age-based \%SPR. \%SPR is expressed as $\mathrm{SPR}_{F=x}$ as a percentage of $\mathrm{SPR}_{F=(0)}{ }^{4} \mathrm{We}$ also compared the value of YPR/W and \%SPR/W to age-based YPR and age-based \%SPR which are generally used.

For Pacific cod off the PCSH, we used von Bertalanffy's growth parameters to calculate weight transition probabilities from Hattori et al. ${ }^{5}$ The weight-length relationship was derived originally (Ueda, unpubl. data, 1998). Natural mortality was set at 0.2 . Weight and age at maturity were set at $3 \mathrm{~kg}$ and 4 yrs. respectively.

For walleye pollock off the PCSH, von Bertalanffy's growth parameters, weight-length relationship, and population number using VPA, were derived from Muroran branch of Hokkaido Hakodate Fisheries Experimental Station. ${ }^{6}$ Natural mortality was set at 0.3 . Weight and age at maturity were set at $400 \mathrm{~g}$ and $3.72 \mathrm{yrs}$. respectively. 


\section{RESULTS}

Fig. 1 shows the YPR/W and age-based YPR, \%SPR/W and age-based \%SPR of Pacific cod off the PCSH. The weight at first capture of $1 \mathrm{~kg}$ is corresponds to the age at first capture of 2.1yrs., and so $3 \mathrm{~kg}$ corresponds to $4.0 \mathrm{yrs}$. The values of $\mathrm{YPR} / \mathrm{W}$ and age-based YPR were parallel at $1 \mathrm{~kg}$ and $2.1 \mathrm{yrs}$, or $3 \mathrm{~kg}$ and $4.0 \mathrm{yrs}$. When weight (age) at first capture are both $1 \mathrm{~kg}$ and $2.1 \mathrm{yrs}$, YPR was the maximum at $F=0.4$. The values of $\%$ SPR/W and age-based $\%$ SPR were slightly different. When $F=0.5$, which was nearly $F=0.48$ in 1997 (estimated using WPA), the differences between the values of \%SPR were 2.1 points ( $1 \mathrm{~kg}-2.1 \mathrm{yrs}$.) and 4.5 points $(3 \mathrm{~kg}-4.0 \mathrm{yrs}$.).
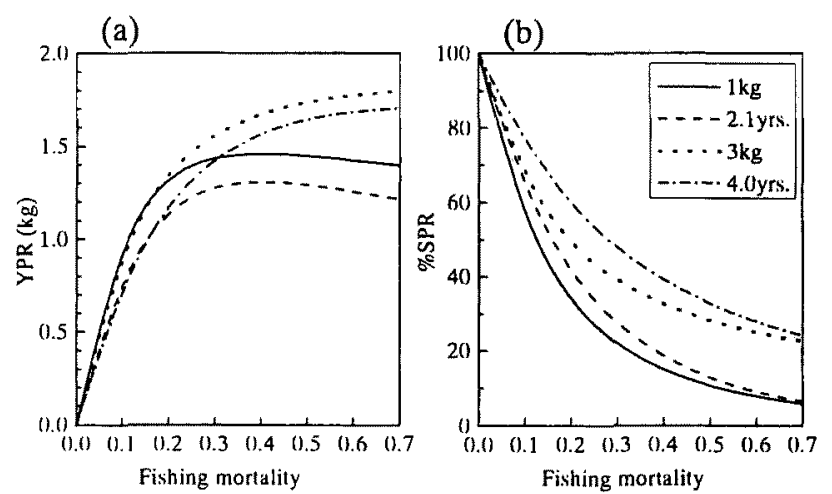

Fig. 1 YPR/W and age-based YPR (a), and \%SPR/W and agebased \%SPR (b) of Pacific cod off the PCSH. (a)

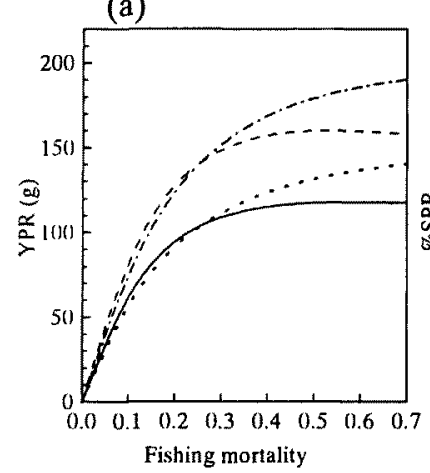

(b)

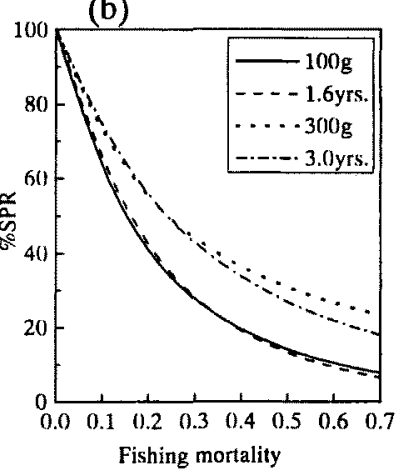

Fig. 2 YPR/W and age-based YPR (a), and \%SPR/W and agebased \%SPR (b) of walleye pollock off the PCSH.

Fig. 2 shows the YPR/W and age-based YPR, and \%SPR/W and age-based \%SPR of walleye pollock off the PCSH. The weight at first capture of $100 \mathrm{~g}$ corresponds to the age at first capture of 1.6yrs., and so $300 \mathrm{~g}$ corresponds to $3.0 \mathrm{yrs}$. The differences between YPR/W and age-based YPR were larger than in the case of Pacific cod, but the values were also parallel for each combination. The values of $\%$ SPR/W and age-based \%SPR were nearly the same. When $F=0.5$, which was nearly $F=0.49$ in 1996 (estimated using WPA), the differences between the values of $\%$ SPR were only 0.9 points $(100 \mathrm{~g}-1.6 \mathrm{yrs}$.) and 4.0 points $(300 \mathrm{~g}-3.0 \mathrm{yrs}$.).

\section{DISCUSSION}

The value of YPR/W and age-based YPR were parallel for Pacific cod and walleye pollock off the PCSH. This result indicates that YPR/W is also suitable for calculation of $F_{\max }$ or $F_{0.1}$, etc. While for Pacific cod, the values of \%SPR/W were slightly smaller than age-based \%SPR, in the case of walleye pollock \%SPR/W and age-based \%SPR were nearly the same. This result indicates the potential of the weight-based method for calculating $F_{\% \text { SPR }}$. We assumed normal distribution of weight in next year. If assume other distribution (log-normal, gamma, etc.) the results of weight-based and age-based methods may different.

There will be some difficulty in determining the variance of weight for constructing $\mathbf{P}$. We examined the sensitivity of this to the result of population estimation, and confirmed that the magnitude of dispersion slightly affects to the estimated population number using WPA. The effect on YPR/W and SPR/W will be potentially small, because YPR/W and SPR/W are calculated from a similar model to WPA.

Using WPA, YPR/W and SPR/W enables to estimate biomass and to assess stock conditions totally based on weight.

\section{REFERENCES}

1. Hilborn R., Walters CJ. Quantitative fisheries stock assessment. Chapman and Hall, London, 1992.

2. Matsuishi T. Development and accuracy assessment of a method of separable VPA based on catch-at-length data. Mem. Fac. Fish. Hokkaido Univ. 1997; 43: 1-80

3. Ueda Y, Matsuishi T, Kanno Y. Weight-based population analysis: an estimation method. Fish. Res. 2001; 50: 271-278.

4. Mace PM. Relationships between common biological reference points used as thresholds and targets of fisheries management strategies. Can. J. Fish. Aquat. Sci. 1994; 51: 110-122.

5. Hattori T, Sakurai Y, Simazaki K. Age determination by sectioning of otoliths and growth pattern of Pacific cod. Nippon Suisan Gakkaishi 1992; 58: 1203-1210.

6. Hokkaido Hakodate Fisheries Experimental Station, Muroran, The posture of Walleye Pollack fishery in the southern Hokkaido part of Pacific Ocean. 1996. (in Japanese) 